\title{
Skin Metastasis from Malignant Ovarian Tumor - A Rare Entity
}

\author{
TS CHOWDHURYa, U RUMAN ${ }^{\mathrm{b}}$, TA CHOWDHURYc
}

\section{Summary:}

Skin metastasis from malignant ovarian carcinoma is rare and seen in only $3 \%$ of patients. Hence, we report a case of ovarian cancer with scar metastasis. The patient, a 31 year old woman presented with two skin nodules in the previous laparotomy scar which revealed metastatic papillary serous

\section{Introduction:}

Ovarian carcinoma is relatively uncommon before 40 years of age. Ovarian epithelial adenocarcinomas rarely metastasize to the skin and can be seen in about 3\% of patients ${ }^{1}$. Skin metastasis may be the first presenting sign of metastatic carcinoma of the ovary .They may resemble the primary tumor in color and consistency and vary in size up to several centimeters. They have diagnostic importance because the prognosis is poor with the median survival of 4 months ${ }^{2}$. However, in selected cases, integrated multi modality treatment may result in palliation of the symptoms and even a prolonged survival.

Here, we report a patient with ovarian cancer with scar metastasis in the form of two skin nodules on each side of previous laparotomy scar and also review of the available literature.

\section{Case Report:}

A 31 years old multiparous, non diabetic and normotensive woman was admitted in BIRDEM hospital

a. Dr. TS Chowdhury, Assistant Professor, Department of Obstetrics and Gynecology, Ibrahim Medical College and BIRDEM Hospital, Dhaka, Bangladesh.

b. Dr. U Ruman, Medical officer, Department of Obstetrics and Gynecology, BIRDEM Hospital, Dhaka, Bangladesh.

c. Prof. TA Chowdhury, Professor and Chief Consultant, Dept of Obstetrics and Gynecology, Ibrahim Medical College and BIRDEM Hospital, Dhaka, Bangladesh.

Address of Correspondence: Dr. Tanjeen Sabina Chowdhury, Room-623, 5th Floor, BIRDEM-2 General Hospital, Shegun Bagicha (opposite to Press Club) Dhaka, Bangladesh, Tel: 01713037853.

Received: 20 October, 2013

Accepted: 9 November, 2014 cyst adenocarcinoma on histopathology. So, it should be considered that ovarian malignancy is not always confined to pelvis and may present with associated skin lesion.

Key words: Ovarian carcinoma, Skin metastasis.

(J Banagladesh Coll Phys Surg 2015; 33: 41-43)

with the complaints of two symmetrical swellings on each end of previous laparotomy scar which were soft, moderately painful and were gradually increasing in size [Fig-1]. She also had occasional moderate lower abdominal pain for previous three months which radiated to groin and to the sub costal area. She gave history of left ovarian cystectomy and appendicectomy two years back but could not produce any document. She had another laparotomy a year later, when another cyst was removed from the left ovary. Histopathology of the tumor revealed a section of ovarian tissue showing a cyst wall lined by single layer of ciliated columnar epithelium. No evidence of malignancy was seen. Diagnosis was benign serous cystadenoma. She was a regularly menstruating woman with moderate dysmenorhoea.

For these swellings, the patient consulted a gynecologist and an ultra sound of whole abdomen was done .It

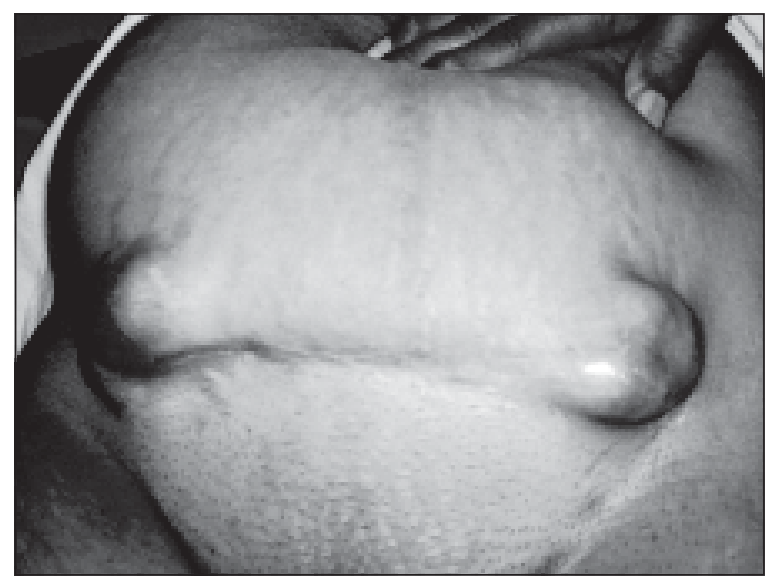

Fig-1: Cutaneous swelling on each end of scar. 
showed mild ascitis, bulky uterus, right adnexal cyst measuring 66X56 mm and a cystic lesion measuring 46X38x38 mm superficially in the scar area. She was referred to BIRDEM hospital for further investigations and management.

On admission, she was mildly anaemic. There were two similar cystic, bluish, moderately tender swellings on each end of laparotomy scar. Surface was irregular and size was $4 \times 5 \mathrm{~cm}$ each. No intra abdominal mass was felt on palpation. Vaginal examination revealed an irregular mass at the upper end of vagina which bled on touch. Serum CA- 125 was more than 500 U/ ml.Ultrasound was repeated on admission which showed marked ascitis with bilateral multi loculated cystic adnexal masses that were in the right adnexal region measuring about $111 \times 50 \mathrm{~mm}$ and in left adnexal region 46x29 mm.Uterus was bulky. It also showed superficial masses in the abdominal wall which were multi loculated and thick walled lesions suggesting the possibility of endometrioma.Other investigations were normal.

Laparotomy was done by Pfannenstiel incision. Two medium sized multiloculated hemorrhagic masses at both ends of previous scar were seen [Fig-2]. There was also a single bluish cystic mass on the anterior rectus sheath. There were two similar cysts on the rectus abdominis muscle. These were removed.

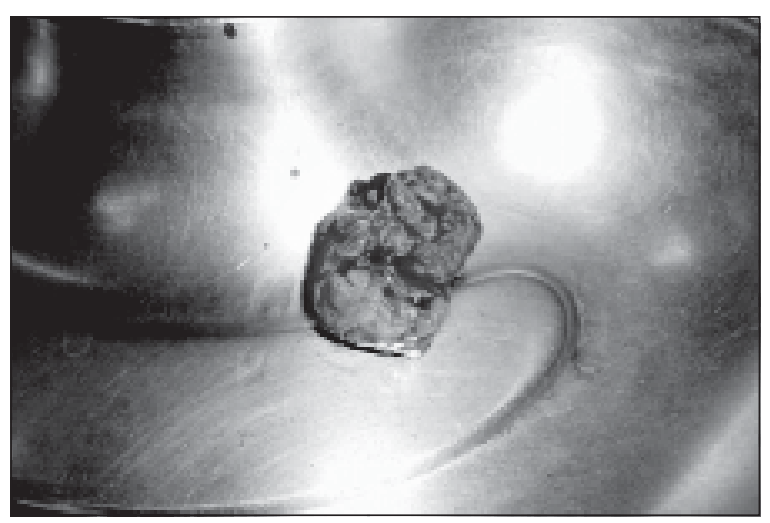

Fig.-2: Cutaneous swelling after excision.

On opening the abdomen, large amount of straw colored fluid came out. Bowels were densely adherent with the pelvic organs. Bilateral malignant ovarian tumors with wide spread metastasis was seen in pelvis. There was formation of omental cake due to extensive metastasis which extended up to the margin of transverse colon. Total abdominal hysterectomy with bilateral salpingo oophorectomy was done. Post operative period was uneventful.

Cytology of peritoneal fluid showed metastatic malignant adenocarcinoma.Tissue from omentum and the gut wall also showed metastatic adeno carcinoma. Sample from the uterus and adnexae showed papillary serous cystadenocarcinoma of ovaries and chronic cervicitis with squamous metaplasia in the uterus.Haemorrhagic mass from skin revealed a metastatic papillary serous cyst adenocarcinoma [Fig-3].

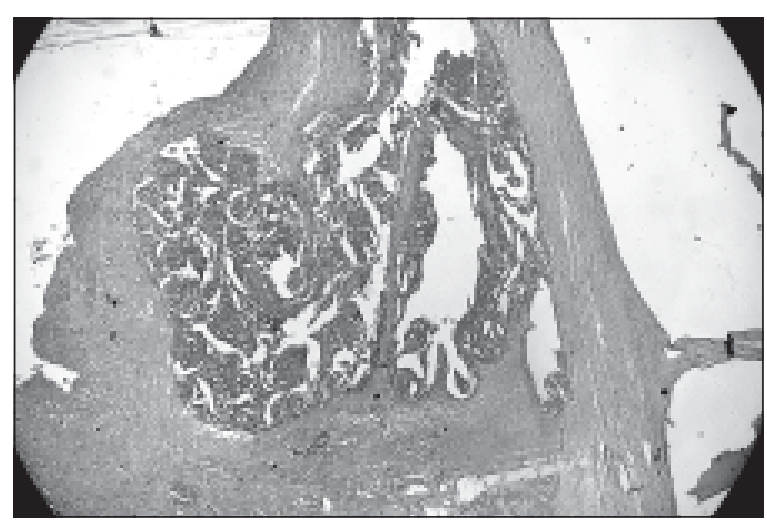

Fig.-3: Histopathology from skin showing metastatic papillary serous cystadenocarcinoma.

The patient was discharged at $10^{\text {th }}$ post operative day with advice to consult an oncologist for further treatment.

\section{Discussion:}

Ovarian carcinoma is a relatively common malignancy of female genital tract. The disease is usually confined to the peritoneal cavity at presentation and throughout its course in approximately $85 \%$ of the patients ${ }^{3}$.The most common mode of spreading ovarian carcinoma to skin is retrograde, from involved proximal lymph nodes. The incidence of cutaneous metastasis from ovarian cancer is estimated to be $3.8 \%{ }^{4}$.It is relatively rare as compared with up to $24 \%$ occurrence with breast carcinoma ${ }^{5}$.The first sign of skin metastasis may be characterized by firm, round or oval, mobile, non-painful nodules. These nodules may be rubbery, firm or hard in texture and vary in size from very small lesion to large tumours. They may be skin colored, red or blue black. Sometimes multiple nodules appear rapidly .These nodules may breakdown and ulcerate through the skin. But this skin metastasis may also present as 
inflammatory lesions or cicatricial plaques. In most patients, however they manifest as cuteneous nodules in the abdominal wall with the usual location being near scar or actually in the scar of the prior surgical intervention ${ }^{6}$. -

In our case, the patient presented with two identical, symmetrical swellings one on each end of previous laparotomy scar.

Skin metastasis from ovarian carcinoma usually manifests in advanced disease with widespread peritoneal involvement and indicate a poor prognosis. The most important prognostic factor associated with survival is the interval between the diagnosis of ovarian cancer and the cuteneous involvement ${ }^{7}$.Mean survival period after diagnosis is 4 months but there are few patients who have survived for many years after the diagnosis $^{8}$.

Metastatic ovarian carcinoma is usually a difficult problem for the clinicians because of its unsatisfactory response to conventional treatment. The skin metastasis is commonly associated with widespread intra peritoneal involvement as we have seen in our case. So, the main approach to the patient should be based on the containment of abdominal disease and surgical resection should be considered.

This patient was treated by total abdominal hysterectomy with bilateral salpingo-oophorectomy with infracolic omentectomy. Surgical enucleation of the skin nodules was done. Later on, we referred her to oncologist for chemotherapy.

For local cuteneous disease, surgical resection should be considered ${ }^{2}$.For extensive cuteneous disease; electro coagulation has been used successfully. Other treatment modalities include liquid nitrogen cryotherapy, photo dynamic therapy, CO2 laser therapy, pulsed dye laser therapy, intra lesional chemotherapy and cytokines.

Cuteneous metastasis from ovarian carcinoma is rare. It is almost always associated with extensive peritoneal disease. But physicians should be aware that ovarian carcinoma is not a disease always confined to the abdomen and the pelvis, so any associated skin swelling should be biopsied.

\section{References:}

1. Perez C A and Law B, Principle and Practice of Radiation Oncology; Ovary, ed: Stambaugh D. $4^{\text {th }}$ ed. Philadelphia: Lippincott Raven. 2004, p 1935- 1936

2. Cormio G, Capotorto M, Vagno G O, Cazzolla A, Carriero C, Selvaggi L. Skin metastasis in ovarian carcinoma: a report of nine cases and a review of the literature. Gynaecolo Oncol. 2003,90: 682-685

3. Powell F C, Cooper A J, Massa M C, Goellner J R, Daniel W P. Sister Joseph's nodule: a clinical and histologic study. $J$ Am Acad Derm. 1984,10: 610-615

4. Krumerman M, Garret R. Carcinoma metastatic. NY State J Med.1977, 77: 1900-1903

5. Krathen R A, Orengo I F, Roben T. Cuteneous metastasis: A Meta analysis of data. South Med J.2003, 96: 164-167

6. Schonmann R, Altaras M, Biran T, et al. Inflammatory skin metastasis from ovarian carcinoma: A case report and reviews of the literature. Gynaecol Oncol.2003, 90: 670-672

7. Cormio G, Rossi C, Cazzolla A, Resta L, Loverro G, Greco P, Selvaggi L. Distant metastasis in ovarian carcinoma. Int $J$ Gynaecol cancer.2003, 13: 125-129

8. Bese T, Demirkiran F, Ilvan S, Sanioglu C, Arvas M, et al. Skin metastasis in ovarian carcinoma. Int $J$ Gynaecol cancer.2006, 16 (suppl 1): 414-418 